\title{
Ein Glitazon kann ein erneutes vaskuläres Ereignis verhindern
}

Fragestellung: Ist der Peroxisom-Proliferator-aktivierte Rezeptor Gamma-Agonist (PPAR-Gamma) Pioglitazon in der Lage, vaskuläre Ereignisse bei Patienten mit Insulinresistenz nach transitorischer ischämischer Attacke (TIA) und Schlaganfall zu verhindern?

Hintergrund: Bis zu 50\% der Patienten, die eine TIA oder einen ischämischen Insult erlitten haben und keinen Typ-2-Diabetes haben, weisen eine Insulinresistenz auf. Diese erhöht das Risiko vaskulärer Ereignisse über Mechanismen wie arterielle Hypertonie, Hyperglykämie, erhöhte Insulinspiegel, Fettstoffwechselstörungen, Störungen der endothelialen Funktionen, Entzündungsreaktionen, erhöhte Plättchenaggregationsneigung und erhöhte Thrombogenität. Neben Diät, Gewichtsreduktion und körperlicher Aktivität werden in diesen Situationen PPARGamma-Agonisten empfohlen, wobei diese bei Patienten nach TIA und Insulinresistenz bisher nicht untersucht wurden.

Patienten und Methodik: Es handelt sich um eine multizentrische doppelblinde randomisierte Studie bei 3.876 Patienten mit Insulinresistenz ohne Diabetes mellitus, die einen ischämischen Insult oder eine TIA erlitten hatten, und die entweder Pio-

Kernan WN, Viscoli CM, Furie KL et al; IRIS Trial Investigators. Pioglitazon after ischemic stroke or transient ischemic attack. N Engl J Med 2016; 374: 1321 -31; doi: 10.1056/NEJMoa1506930 glitazon $45 \mathrm{mg} /$ Tag oder Placebo erhielten. Der primäre Endpunkt der Studie waren Schlaganfall und Myokardinfarkt.

Ergebnisse: In die Studie wurden 3.895 Patienten ein- geschlossen. Das mittlere Alter betrug 63 Jahre. $88 \%$ der Studienteilnehmer hatten einen Schlaganfall erlitten. Fast alle $\mathrm{Pa}-$ tienten wurden mit Statinen oder Thrombozytenfunktionshemmern behandelt. Die Hälfte erhielt einen ACE-Hemmer oder ein Sartan. Nach einer mittleren Beobachtungszeit von 4,8 Jahren hatten 175 von 1.939 Patienten (9\%) in der PioglitazonGruppe und 228 von 1.937 Patienten $(11,8 \%)$ in der Placebogruppe den primären Endpunkt erreicht. Das entspricht einer Hazard Ratio von 0,76 und damit einer Risikoreduktion von $24 \%(\mathrm{p}=0,007)$.

In der Pioglitazon-Gruppe entwickelten 73 Patienten einen manifesten Diabetes mellitus, in der Placebogruppe dagegen 149 Patienten (3,8\% vs. 7,7\%). Das entspricht einer Hazard Ratio von 0,48 und ein P-Wert von 0,001. Es ergab sich kein Unterschied in der Sterblichkeit.

Im Bereich der Nebenwirkungen führte Pioglitazon signifikant häufiger zu einer Gewichtszunahme über 4,5 kg (52\% vs. $34 \%$ ), Ödemen (35\% vs. $25 \%$ ) und knöchernen Frakturen (5\% vs. $3 \%$ ). Blasentumore waren in der Verumgruppe nicht häufiger als in der Placebogruppe.

Schlussfolgerungen: Bei Patienten ohne Diabetes mellitus, aber mit Insulinresistenz, die einen ischämischen Insult oder eine TIA erlitten haben, ist unter einer Therapie mit $45 \mathrm{mg}$ täglich Pioglitazon im Vergleich zu Placebo das Risiko eines Schlaganfalls oder eines Myokardinfarkts signifikant geringer als in der Placebogruppe. Pioglitazon erniedrigt auch das Risiko, an einem Diabetes mellitus zu erkranken. Unter Pioglitazon kommt es häufiger zu Gewichtszunahme, Ödemen und knöchernen Frakturen.

\section{- Kommentar von Hans-Christoph Diener, Essen}

\section{Nicht medikamentöse Maßnahmen stehen an erster Stelle}

Die Ergebnisse dieser Studie sind sehr schwer in den klinischen Alltag zu integrieren. Insgesamt haben Studien zu Pioglitazon in der Vergangenheit bei Patienten mit Diabetes mellitus nicht belegen können, dass sie vaskuläre Endpunkte wie Schlaganfall und Herzinfarkt reduzieren. Das ist die erste Endpunktstudie bei Patienten, die eine TIA oder einen Schlaganfall erlitten haben und unter einer Insulinresistenz leiden, bei denen ein Benefit bezüglich Schlaganfall und Herzinfarkt beobachtet wurde. Diesem Nutzen stehen allerdings nicht unerhebliche Nebenwirkungen gegenüber wie Gewichtszunahme, Ödeme und insbesondere knöcherne Frakturen. In früheren Zulassungsstudien zu Pioglitazon bei Diabetikern war auch eine signifikante Erhöhung von malignen Blasentumoren gefunden worden, was dazu führte, dass die Substanz in Deutschland kaum mehr eingesetzt wird. Bei sorgfältiger Güterabwä- gung zwischen Nutzen und Risiko würde man sicher nicht medikamentösen Maßnahmen zur Behandlung der Insulinresistenz wie Gewichtsreduktion, Sport und Diät den Vorrang geben.

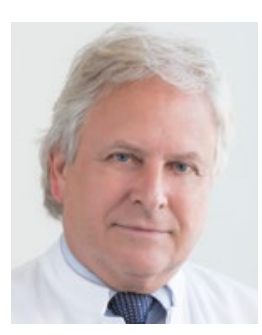

Prof. Dr. med. Hans-Christoph Diener, Essen

Direktor der Klinik für Neurologie,

Universitätsklinikum Essen

E-Mail:h.diener@uni-essen.de 